\title{
Chest Pain
}

National Cancer Institute

\section{Source}

National Cancer Institute. Chest Pain. NCI Thesaurus. Code C38665.

Pain in the chest. 